\title{
PREVALÊNCIA DE COMPLICAÇÕES MICRO E MACROVASCULARES E DE SEUS FATORES DE RISCO EM PACIENTES COM DIABETES MELITO DO TIPO 2 EM ATENDIMENTO AMBULATORIAL
}

\author{
Rafael Selbach Scheffel, Desirê Bortolanza, Cristiane Seganfredo Weber, luciana Abarno da Costa, \\ Luís Henrique Canani, Kátia Gonçalves dos Santos, Daisy Crispim, Israel Roisenberg, \\ Hugo Roberto Kurtz lisbôa, Glaucia Sarturi Tres, Balduíno Tschiedel, Jorge luiz Gross* \\ Trabalho realizado no Serviço de Endocrinologia do Hospital de \\ Clínicas de Porto Alegre, Porto Alegre, RS.
}

RESUMO - OBjetivos. 0 diabetes melito (DM) do tipo 2 está associado ao desenvolvimento de complicações macroangiopáticas [cardiopatia isquêmica (Cl), doença vascular periférica (DVP) e acidente vascular cerebral (AVC)] e microangiopáticas [retinopatia diabética (RD), nefropatia diabética (ND) e neuropatia sensitiva distal (NSD)]. Os objetivos deste estudo foram avaliar a prevalência das complicações crônicas em pacientes com DM do tipo 2 e aferir os seus possíveis fatores de risco.

MétODos. Estudo transversal, incluindo 927 pacientes com DM do tipo 2 atendidos ambulatorialmente em três centros médicos do Rio Grande do Sul: Hospital de Clínicas de Porto Alegre ( $n=$ 475), Grupo Hospitalar Conceição $(n=229)$ e Hospital São Vicente de Paula $(n=223)$. Dentre os pacientes, $42 \%$ eram homens, a média de idade era de $59 \pm 10$ anos e a mediana da duração do DM do tipo 2 era II (5 - 43) anos. A RD foi definida por fundoscopia direta; a $\mathrm{Cl}$ através do questionário da $\mathrm{OMS}$ e/ou alterações eletrocardiográficas e/ou anormalidades perfusionais na cintilografia miocárdica; a NSD por sintomas compatíveis e ausência de sensação ao monofilamento de $10 \mathrm{~g}$ e/ou ao diapasão; a DVP pela claudicação e ausência de pulsos pediosos; o AVC por seqüelas ou história compatível e a ND pela excreção urinária de albumina ( $\geq 20 \mu \mathrm{g} / \mathrm{min}$ ). A hipertensão arterial sistêmica (HAS) foi definida pelos níveis pressóricos ( $\geq 140 / 90 \mathrm{mmHg}$ ) e/ou uso de drogas anti-hipertensivas. Foram calculados 0 índice de massa corporal (IMC, $\mathbf{~ g g} / \mathrm{m}^{2}$ ) e a razão cintura-quadril (RCQ).

Resultados. A Cl estava presente em $36 \%$ e a DVP em $33 \%$ dos pacientes. Dentre as complicações microvasculares, $37 \%$ tinham doença renal (I2\% macroalbuminúricos) e $48 \%$ RD (I5\% retinopatia proliferativa). A NSD foi encontrada em $36 \%$ dos pacientes. HAS estava presente em $73 \%$ dos pacientes. 0 colesterol estava acima de $200 \mathrm{mg} / \mathrm{dl} \mathrm{em} \mathrm{64 \% ,} \mathrm{enquanto} \mathrm{o} \mathrm{IMC} \mathrm{>}$ $30 \mathrm{~kg} / \mathrm{m}^{2}$ em $36 \%$. Vinte e dois por cento dos pacientes eram fumantes atuais e $21 \%$ ex-tabagistas.

Conclusáo. As complicações crônicas do DM do tipo 2 têm uma alta prevalência nos pacientes ambulatoriais de hospitais gerais. Praticamente todos os pacientes apresentavam pelo menos um fator de risco para doença cardiovascular, o que justifica o seu rastreamento e controle.

UnItermos: Diabetes melito. Complicações crônicas. Fatores de risco.

\section{INTRODUÇÃO}

O comprometimento ateroesclerótico das artérias coronarianas, dos membros inferiores e das cerebrais é comum nos pacientes com diabetes melito (DM) do tipo 2 e constitui a principal causa de morte destes pacientes. Estas complicações macroangiopáticas podem ocorrer mesmo em estágios precoces do DM e se apresentam de forma mais difusa e grave do que em pessoas sem DM'. Além disso, pacientes com DM podem apresentar problemas de

* Correspondência: Rua Ramiro Barcelos, 2350 - Prédio 12 - 4 andar CEP: $90035-003$ - Porto Alegre - RS - Brasil Fone/fax (51) 33325188 visão ${ }^{2}$, doença renal [nefropatia diabética $(\mathrm{ND})]^{3}$ e dano neuronal [neuropatia sensitiva distal (NSD) $]^{4}$, que são chamadas de complicações microangiopáticas.

A freqüência das complicações crônicas do DM do tipo 2 varia de acordo com as populações estudadas. Os pacientes com DM do tipo 2 têm uma propensão duas a quatro vezes maior de morrer por doença cardíaca em relação a não diabéticos, e quatro vezes mais chance de ter doença vascular periférica (DVP) e acidente vascular cerebral (AVC) $)^{5-10}$. O DM do tipo 2 também é apontado como uma das principais causas de cegueira entre adultos com idade de 20 a 74 anos 2,11 . Em alguns levantamentos, após 15 anos do diagnóstico de DM do tipo 2, a retinopatia diabética (RD) esteve presente em $97 \%$ dos usuários de insulina e em $80 \%$ dos não usuários ". A prevalência de ND varia de $10 \%$ a $40 \% 3,12$ e a de NSD, de $60 \%$ a $70 \%{ }^{4}$

Entre os fatores envolvidos na etiologia das complicações crônicas do DM do tipo 2, destacam-se a hiperglicemia, a hipertensão arterial sistêmica, a dislipidemia e o tabagismo ${ }^{13,15}$. Além destes, outros fatores de risco não convencionais têm sido descritos: disfunção endotelial, estado pré-trombótico e inflamação ${ }^{16}$.

Os objetivos deste estudo foram avaliar a prevalência das complicações crônicas do diabetes em pacientes com DM do tipo 2, em atendimento ambulatorial de três centros médicos do Rio Grande do Sul, e aferir os fatores de risco relacionados com a etiologia destas complicações. 


\section{Métodos}

Foi realizado um estudo transversal incluindo 927 pacientes com DM do tipo 2 atendidos ambulatorialmente em três centros médicos do Rio Grande do Sul. Estes centros foram os Serviços de Endocrinologia do Hospital de Clínicas de Porto Alegre (HCPA, $n=475)$, do Grupo Hospitalar Conceição, em Porto Alegre (GHC, $n=229)$ e do Hospital São Vicente de Paula, em Passo Fundo (HSVP, $n=223$ ). Estes centros fazem parte de um estudo multicêntrico regional iniciado em 2001. O protocolo foi aprovado pelo comitê de ética local. Os pacientes foram avaliados sistematicamente em relação à presença das seguintes complicações: ND, RD, cardiopatia isquêmica (CI), DVP e AVC. Os pacientes com insuficiência renal crônica (creatinina $\geq 2 \mathrm{mg} / \mathrm{dl}$ ) e aqueles atendidos nas unidades de diálise foram analisados em separado. DM do tipo 2 foi diagnosticado de acordo com as recomendações da OMS ausência de episódios prévios de cetoacidose diabética, diagnóstico de DM após 35 anos de idade e necessidade de uso de insulina somente após dois anos de diagnóstico ${ }^{17}$.

Os pacientes responderam a um questionário padronizado incluindo idade, tempo de diagnóstico de diabetes, hábito de fumar e medicações em uso. O exame físico e a avaliação laboratorial foram realizados com a finalidade de avaliar as complicações crônicas e os fatores de riscos para as mesmas. A medida de peso e altura foi feita em balança antropométrica, sem sapatos e com roupas leves. $\mathrm{O}$ índice de massa corporal (IMC) foi calculado pela razão peso $(\mathrm{kg}) /$ altura $^{2}\left(\mathrm{~m}^{2}\right)$. A circunferência da cintura (na altura da cicatriz umbilical) e do quadril (parte mais larga) foram medidas e o índice cintura/quadril (ICQ) foi calculado. A pressão arterial sistêmica foi medida duas vezes com intervalo de 10 minutos na posição sentada, no membro superior esquerdo, com esfigmomanômetro de coluna de mercúrio (fases I e $V$ dos ruídos de Korotkoff). A presença de hipertensão foi considerada quando a pressão arterial fosse $\geq 140 / 90 \mathrm{mmHg}$ e/ou em uso de drogas anti-hipertensivas. A avaliação da presença de neuropatia foi realizada através da pesquisa de reflexos tendinosos profundos (patelar e aquileu) e avaliação da sensibilidade vibratória (diapasão de 128 ciclos por segundo no hálux) e tátil (monofilamento de $10 \mathrm{~g}$ em três locais dos pés).

\section{Identificação das complicações microangiopáticas}

A presença de RD foi avaliada através da fundoscopia direta após dilatação pupilar e foi classificada como: I) sem sinais de RD; 2) RD não-proliferativa (microaneurismas, hemorragia, exsudatos duros); ou 3) RD proliferativa (presença de neovasos e/ou tecido fibroso na cavidade vítrea). A ND foi definida por aumento na excreção urinária de albumina (EUA) na ausência de infecção urinária ou outras anormalidades renais. A ND foi dividida em microalbuminúria (EUA 20-200 $\mu \mathrm{g} / \mathrm{min}$ ) e macroalbuminúria (EUA > $200 \mu \mathrm{g} / \mathrm{min}$ ).

A presença de NSD foi definida por sintomas e ausência de reflexos tendinosos profundos e/ou ausência da sensibilidade vibratória e/ ou ao monofilamento nos membros inferiores.

\section{Identificação das complicações macroangiopáticas}

Estas complicações foram avaliadas somente nos centros do Hospital de Clínicas de Porto Alegre e São Vicente de Passo Fundo $(n=698)$. A DVP foi diagnosticada quando da presença de claudicação intermitente [questionário cardiovascular da Organização Mundial da Saúde $(O M S)^{18}$ ] ou diminuição/ausência de pulsos pediosos ao exame físico. A presença de $\mathrm{Cl}$ foi baseada na presença de angina ou possível infarto de acordo com questionário cardiovascular da OMS, e/ou presença de alterações eletrocardiográficas (código Minnesota) $)^{19}$ e/ou presença de anormalidades perfusionais (fixas ou variáveis) no exame de cintilografia miocárdica em repouso e após dipiridamol. Todos os pacientes com avaliação para $\mathrm{Cl}$ realizaram cintilografia miocárdica. A presença de AVC foi estabelecida quando havia relato prévio e/ou presença de achados compatíveis (seqüelas).

\section{Avaliação laboratorial}

A EUA foi medida através da técnica de imunoturbidimetria em amostras de urina de 24 horas com tempo marcado (três amostras por paciente com 15 dias de intervalo, em urina estéril). A presença de microalbuminúria ou macroalbuminúria foi confirmada por pelo menos duas medidas com intervalo de três a seis meses.

Os níveis de glicose foram determinados pelo método glicose oxidase, a creatinina pela reação de Jaffé, a glico hemoglobina $\left(\mathrm{HbA}_{1 c}\right)$ por um processo HPLC - troca iônica (valores de referência: 2,7 - 4,3\%); os triglicerídeos e os níveis de colesterol por métodos enzimáticos. O LDL-colesterol foi calculado pela equação de Friedewald.

\section{Análise dos dados}

Os dados foram descritos por média \pm desvio padrão (DP) ou números absolutos e percentuais. As prevalências foram comparadas através do teste do qui-quadrado. Foi considerado significativo um alfa menor ou igual a $5 \%$.

\section{Resultados}

As características clínicas e laboratoriais dos pacientes ambulatoriais encontram-se na Tabela I. Foram analisados 927 pacientes com média de idade de $59 \pm 10$ anos, sendo 390 do sexo masculino e 537 do sexo feminino. Os pacientes apresentavam uma mediana de duração de DM de II anos (com variação de 5 a 43 anos), sendo que a maioria utilizava hipoglicemiantes orais (52\%) para o tratamento. Insulina isoladamente era usada por $28 \%$ dos pacientes e insulina em combinação com hipoglicemiantes orais por $7 \%$. A dieta era o único tratamento em $13 \%$ dos pacientes.

Em relação ao controle metabólico, apenas 16\% e 7\% dos pacientes apresentavam valores ideais de glicemia de jejum ( $\leq 110 \mathrm{mg} /$ dl) e de HbAlc $(\leq 4,3 \%)$, respectivamente. Valores de controle metabólico aceitáveis (glicemia de jejum $\leq 126 \mathrm{mg} / \mathrm{dl} \mathrm{e} \mathrm{HbAlc}$ $\leq 5,3 \%$ ) foram encontrados em $23 \%$ e $25 \%$ dos pacientes, respectivamente.

A Figura I mostra a prevalência dos fatores de risco cardiovasculares nos pacientes estudados, destacando-se a hipertensão (73\%), os valores de colesterol acima de $200 \mathrm{mg} / \mathrm{dl}$ (64\%), o IMC > $30 \mathrm{~kg} / \mathrm{m}^{2}(36 \%)$ e o ICQ > 0,9 para homens e $>0,85$ para mulheres (88\%). Vinte e dois por cento dos pacientes eram fumantes atuais e $21 \%$ ex-tabagistas.

A prevalência de complicações crônicas dos pacientes é mostrada na Figura 2. Das complicações macrovasculares, as mais freqüentes foram Cl (36\%) e DVP (33\%). Dentre as microvasculares, $37 \%$ tinham doença renal, sendo que $12 \%$ eram macroalbu minúricos; $48 \%$ apresentavam RD, dos quais 15\% tinham retinopatia proliferativa. A NSD foi avaliada somente nos pacientes prove nientes dos centros do HCPA e do HSVP 


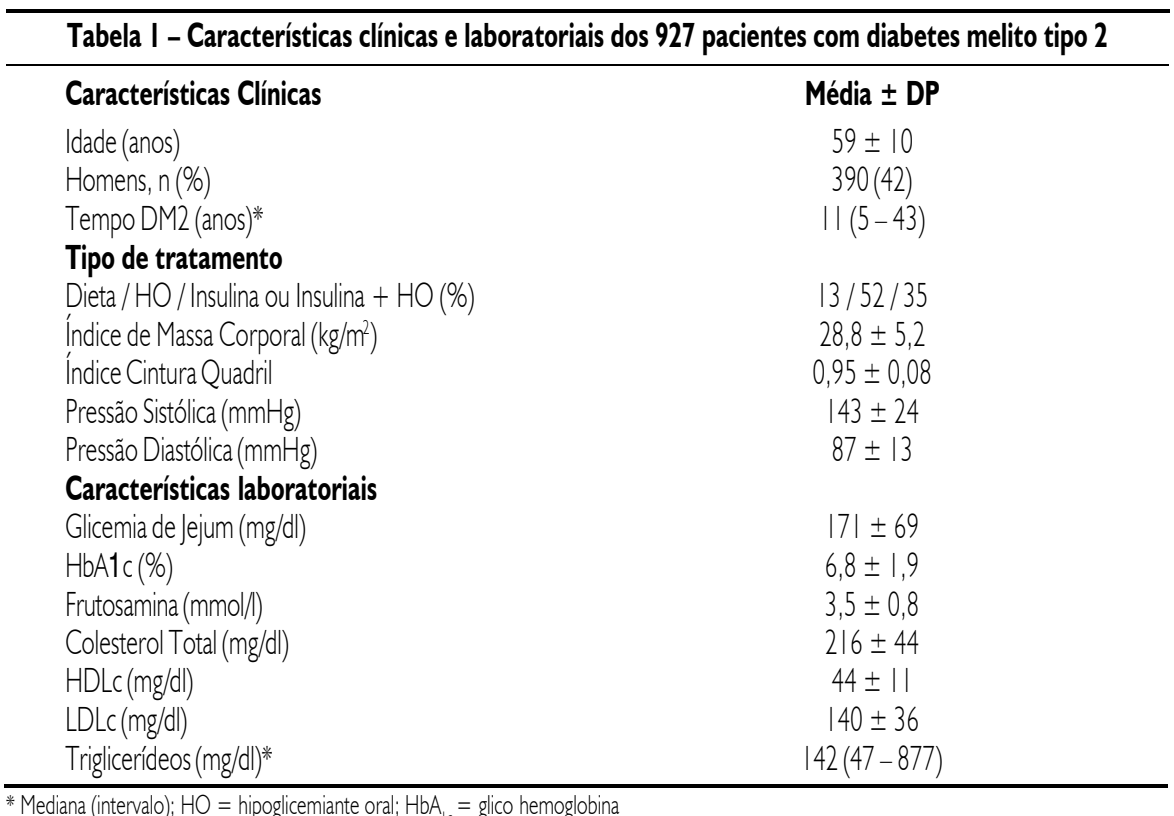

Figura I - Prevalência de fatores de risco cardiovasculares

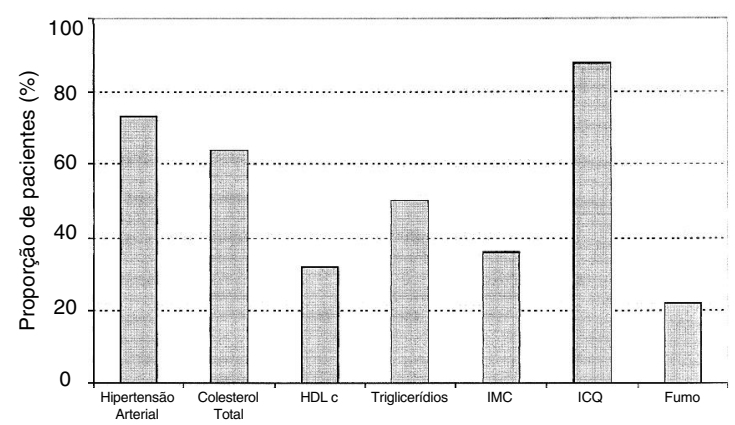

Colesterol total $\geq 200 \mathrm{mg} / \mathrm{dl} ; \mathrm{HDLc}<35 \mathrm{mg} / \mathrm{dl}$ para homens e $<39 \mathrm{mg} / \mathrm{dl}$ para mulheres; triglicerídios $\geq 150 \mathrm{mg} / \mathrm{dl} ; \mathrm{IMC}$ (índice de massa corporal) $>30 \mathrm{~km} / \mathrm{m}^{2}$; ICQ (índice cintura quadril) $>0,9$ para homens e $>0,85$ para mulheres

Figura 2 - Prevalência das complicações crônicas do diabetes melito do tipo 2

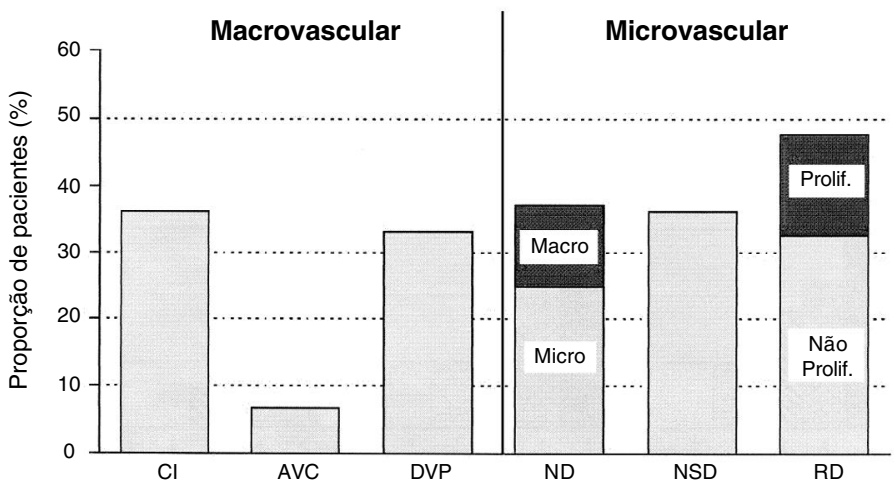

$\mathrm{Cl}$ = cardiopatia isquêmica; $\mathrm{AVC}=$ acidente vascular cerebral; $\mathrm{DVP}=$ doença vascular periférica; $\mathrm{ND}$ = nefropatia diabética; NSD = neuropatia sensitiva distal; $R D=$ retinopatia diabética $(n=698)$ e estava presente em $36 \%$ dos pacientes. A prevalência das complicações é tradicionalmente associada com a duração do DM. Entretanto, mesmo entre os pacientes com pouco tempo conhecido de DM ( $<5$ anos), uma substancial proporção dos pacientes apresentou RD (27\%), ND (28\%), NSD (22\%), Cl (26\%) e DVP (23\%).

Menos de $1 \%$ dos pacientes apresentaram somente um fator de risco isolado (hipertensão ou dislipidemia ou mau controle metabólico ou obesidade ou microalbuminúria ou tabagismo). Aproximadamente 5\% apresentaram dois fatores, enquanto $27 \%$ e $43 \%$ apresentaram três ou quatro, respectivamente. Cinco fatores estavam presentes em 23\% dos pacientes.

Dos 117 pacientes com nefropatia avançada, 81 foram identificados nas unidades de tratamento dialítico e 36 tinham insufi ciência renal crônica (creatinina sérica $\geq 2$ $\mathrm{mg} / \mathrm{dl}$ ). A idade média destes pacientes foi $60 \pm 9$ anos e o tempo conhecido foi de DM de $16 \pm 9$ anos. Os homens representavam $67 \%$ do grupo. Os dados de lipídeos séricos e HbA1c estavam disponíveis somente para 5 I destes pacientes. Dezoito por cento apresentavam glicemia de jejum em níveis ideais e 13\% valores de $\mathrm{HbA1}$ c nesta faixa. Além disso, 28\% apresentavam valores aceitáveis para a glicemia de jejum e 47\% valores aceitáveis para a HbA1c. Em relação ao perfil lipídico, 67\% apresentavam colesterol total acima de $200 \mathrm{mg} / \mathrm{dl}, 65 \%$ triglicerídeos > $150 \mathrm{mg} / \mathrm{dl}$ e $47 \%$ apresentavam HDL colesterol baixo. A obesidade $\left(\right.$ IMC $\left.>30 \mathrm{~kg} / \mathrm{m}^{2}\right)$ estava presente em $20 \%$ dos pacientes.

Observou-se um aumento linear da freqüência das complicações microangiopáticas de acordo com o número de fatores de risco presentes (Figura 3). Entre os pacientes com um ou dois fatores de risco nenhum apresentava ND. Entre os pacientes com três, quatro ou cinco fatores, a presença de ND foi de aproximadamente $12 \%$, $27 \%$ e $98 \%$ $(p<0,01)$. O mesmo padrão foi observado para a presença de RD e NSD. A prevalência de RD e NSD entre os pacientes com um, dois, três, quatro ou cinco fatores de risco associados foi 10\%, 18\%, 29\%, 40\%, 65\% e $0 \%, 20 \%, 35 \%, 44 \%, 60 \%$, respectivamente $(p<0,05)$. Em relação às complicações 
Figura 3 - Frequiência das complicações micro angiopáticas de acordo com o número de fatores de risco cardiovasculares

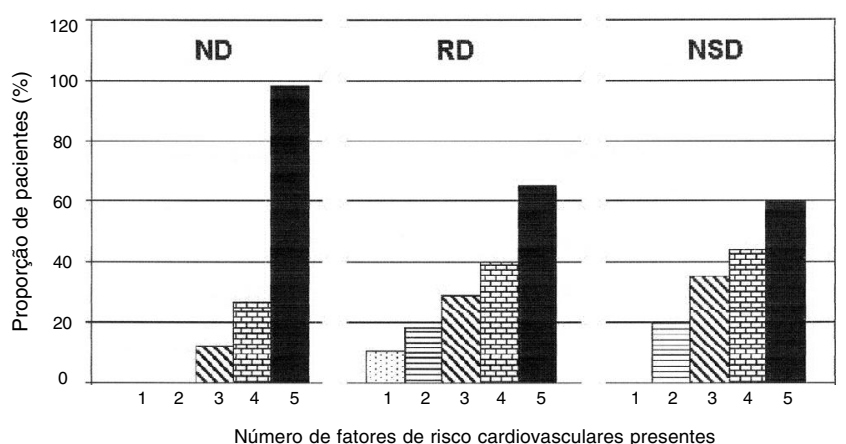

$\mathrm{ND}=$ nefropatia diabética; $R D$ = retinopatia diabética; $N S D$ = neuropatia sensitiva distal

macroangiopáticas (avaliadas somente nos centros do HCPA e HSVP), o mesmo foi observado somente para a presença de AVC. Observou-se um pequeno aumento na prevalência de Cl e DVP com o aumento de fatores de risco cardiovasculares associados, porém, sem atingir significância estatística $(p>0,05)$.

\section{Discussão}

O presente estudo demonstra uma importante prevalência das complicações crônicas nos pacientes com DM do tipo 2 atendidos nos serviços de endocrinologia de hospitais gerais. A prevalência destas complicações não foi desprezível mesmo entre os pacientes com pouco tempo conhecido de DM. Isto reforça a importância da realização de estadiamento de rotina das complicações do DM, mesmo nos pacientes recentemente diagnosticados.

Estudos de prevalência das complicações crônicas do DM com base populacional não estão disponíveis no Brasil. Apesar de ser um hospital geral, o Hospital de Clínicas de Porto Alegre é um centro de referência para manejo de pacientes com DM. Isso pode levar a um aumento da prevalência das complicações nos pacientes atendidos neste centro. Na realidade, em relação às complicações microangiopáticas, a prevalência de RD foi maior entre os pacientes atendidos no HCPA e no GHC do que entre os pacientes atendidos no HSVP ( $50 \%$ e $54 \%$ vs. $35 \%$, p < 0,05). A prevalência de ND foi mais alta entre os pacientes atendidos no HCPA, intermediária nos atendidos no GHC e menor nos atendidos no HSVP (4I\% vs. $36 \%$ vs. $30 \%, p<0,05)$.
Parte dos pacientes avaliados no presente estudo fazem parte de uma coorte de pacientes avaliados no HCPA em 199220. No ano em questão, um total de 212 pacientes com DM do tipo 2 foram avaliados. No presente estudo, analisando-se somente os pacientes atendidos no HCPA, observou-se um aumento na freqüência de $\mathrm{Cl}$ (45\% vs. 25\%) e DVP (32\% vs. $25 \%$ ) em relação ao levantamento de 1992. Por outro lado, no presente levantamento, a RD esteve presente em 50\% dos pacientes em comparação com $51 \%$ no estudo prévio. A prevalência de AVC, NSD e ND foi semelhante nos dois levantamentos. É interessante ressaltar que os pacientes incluídos nos dois estudos eram semelhantes quanto à idade, ao tempo médio conhecido de DM, à proporçãa de homens, à de pacientes hipertensos e de obesos. Também é interessante a comparação com um levantamento realizado em 1982 no mesmo serviço de Endocrinologia do HCPA ${ }^{21}$. Estes três estudos sugerem que a prevalência da ND, da NSD e do AVC parecem ter diminuído de 1982 para 1992 e mantiveram-se estáveis entre 1992 e o presente estudo. Por outro lado, a RD parece estar diminuindo progressivamente $(65 \%$ vs. $51 \%$ vs. $50 \%)$.

\section{Conclusões}

Este levantamento mostra que existe uma diferença na prevalência das complicações do DM de acordo com o local avaliado, sugerindo que possa existir um viés de seleção em centros mais especializados no manejo de pacientes com DM, e reforçando a necessidade de estudos com base populacional para o planejamento de políticas de saúde. Também demonstra que algumas das complicações crônicas estão diminuindo, podendo refletir um melhor tratamento dos fatores de risco como a hiperglicemia e a hipertensão arterial. Por outro lado, as demais complicações mantiveram-se inalteradas independentemente da possível melhora no manejo destes pacientes, sugerindo que outras medidas se fazem necessárias.

\section{Conflito de interesse: não há.}

\section{SUMMARY}

Prevalence of micro and macroanGIOPATIC CHRONIC COMPLICATIONS AND THEIR RISK FACTORS IN THE CARE OF OUT PATIENTS WITH TYPE 2 DIABETES MELLITUS

BACKGROUND. Type 2 diabetes (DM2) has been related to the development of macroangiopatic [coronary heart disease (CHD), peripheral vascular disease (PVD) and stroke] and microangiopatic [retinopathy, nephropathy, and distal sensory neuropathy (DSN)] complications. The aims of this study were to analyze prevalence of complications in DM2 patients and to estimate their associated risk factors.

Methods. Cross-sectional study, including 927 out patients with DM2 from three medical centers in Rio Grande do Sul: Hospital de Clínicas de Porto Alegre $(n=475)$, Grupo Hospitalar Conceição $(n=229)$ and Hospital São Vicente de Paula $(n=223)$. Of the patients $42 \%$ were male, mean age was $59 \pm 10$ years and the median known duration of DM2 was I I (5-43) years. Retinopathy was identified by direct fundoscopy; $\mathrm{CHD}$ by WHO questionnaire and/or abnormal ECG and/or perfusion abnormalities on myocardial scintigraphy; DSN by compatible symptoms and absent sensation on $10 \mathrm{~g}$ monofilament and/or tune fork; PVD by the presence of claudication and absent foot pulses; stroke by presence of sequels and history; and nephropathy by the urinary albumin excretion rate $(\geq 20 \mu \mathrm{g} / \mathrm{min})$. Hypertension was defined by blood pressure ( $\geq 140 / 90 \mathrm{mmHg}$ ) and/or use of antihypertensive drugs. Body mass index (BMI, $\mathrm{kg} / \mathrm{m}^{2}$ ) and waist-to-hip ratio (WHR) were calculated.

REsULTS. CHD was present in $36 \%$ and PVD in $33 \%$ of the patients. Among the microvascular, 37\% had nephropathy (I2\% 
with macroalbuminuria); $48 \%$ retinopathy ( $15 \%$ proliferative retinopathy). DSN was present in 36\%. Seventy three percent of the patients presented arterial hypertension. Cholesterol levels were $>200 \mathrm{mg} / \mathrm{dl}$ in 64\% and $\mathrm{BMI}>30 \mathrm{~kg} / \mathrm{m}^{2}$ in $36 \%$. Twenty two percent of patients were smokers and $21 \%$ ex-smokers.

CONCLUSION. Diabetic complications are frequent among out patients referring to general hospitals. Almost all patients presented at least one risk factor for cardiovascular disease, justifying the efforts for identification and adequate control. [Rev Assoc Med Bras 2004; 50(3): 263-7]

KEY wOrds: Diabetes mellitus. Chronic complications. Risk factors.

\section{ReferênCIAS}

1. American Diabetes Association. Diabetes complications. In: Diabetes 200 I vital statistics. Virginia: Port City Press, Inc; 200 I. p.4374

2. Centers for Disease Control and Prevention. Blindness caused by diabetes - Massachusetts, 1987-1994. JAMA | 996; 276: | 865-86.

3. Gabir MM, Hanson RL, Dabelea D, Impertore G, Rouman J, Bennett PH et al. Plasma glucose and prediction of microvascular disease and mortality: evaluation of 1997 American Diabetes Association and 1999 World Health Organization criteria for diagnosis of diabetes. Diabetes Care 2000; 23: I I 13-8.

4. Franklin GM, Kahn LB, Baxter J, Marshall JA, Hamman RF. Sensory neuropathy in non- insulin-dependent diabetes mellitus. The San Luis Valley Diabetes Study. Am J Epidemiol 1990; 131:633-43.

5. Center for Disease Control and Prevention. Diabetes-related amputations of lower extremities in the Medicare population-Minnesota, 1993-1995. MMWR Morb Mortal Wkly Rep 1998; 47:649-52.

6. Brand FN, Abbott RD, Kannel WB. Diabetes, intermittent claudication, and risk of cardiovascular events. The Framingham Study. Diabetes 1989; 38:504-9.

7. Gu K, Cowie CC, Harris MI. Mortality in adults with and without diabetes in a national cohort of the U.S. population, 1971-1993. Diabetes Care 1998; 21:1138-45.

8. Haffner SM. Coronary heart disease in patients with diabetes. N Engl J Med 2000; 342: 1040-2.

9. Hayden JM, Reaven PD. Cardiovascular disease in diabetes mellitus type 2: a potential role for novel cardiovascular risk factors. Curr Opin Lipidol 2000; 1 1:519-28.

10. Kanters SD, Banga JD, Stolk RP, Algra A. Incidence and determinants of mortality and cardiovascular events in diabetes mellitus: a meta-analysis. Vasc Med 1999; 4:67-75.

II. Center for Disease Control and Prevention. Blindness caused by diabetes-Massachusetts, 1987-1994. MMWR Morb Mortal Wkly Rep |996; 45:937-41.

12. Mogensen CE, Christensen CK, Vittinghus $E$. The stages in diabetic renal disease with emphasis on the stage of incipient diabetic nephropathy. Diabetes 1983; 32(Suppl 2):64-78.

13. Stamler J, Vaccaro O, Neaton JD, Wentworth D. Diabetes, other risk factors, and 12-yr cardiovascular mortality for men screened in the Multiple Risk Factor Intervention Trial. Diabetes Care 1993; 16:434-44.
14. Wolf PA, D'Agostino RB, Belanger AJ, Kannel WB. Probability of stroke: a risk profile from the Framingham Study. Stroke 199|; 22:312-8.

15. Diabetes Control and Complications Trial Research Group. The effect of intensive treatment of diabetes on the development and progression of long-term complications in insulin-dependent diabetes mellitus. N Engl J Med 1993; 329:977-86.

16. Saito I, Folsom AR, Brancati FL, Duncan BB, Chambless LE, McGovern PG. Nontraditional risk factors for coronary heart disease incidence among persons with diabetes: the Atherosclerosis Risk in Communities (ARIC) Study. Ann Intern Med 2000; 133:81-91.

17. WHO Study Group. Diabetes mellitus. Geneve: World Health Organization; 1985. (Technical Report Series 727)

18. Rose GA. The diagnosis of ischemic heart pain and intermittent claudication in field surveys. Bull World Health Organ 1962; 27:654-8.

19. Rose GA, Blackburn H, Gillum RF, Prineas RJ. Cardiovascular survey methods. 2nd ed. World Health Organization Bulletin; 1982. p. I 23-43.

20. Beck MO. Prevalência de complicações crônicas e fatores de risco para nefropatia diabética em pacientes com diabete melito tipo II [dissertação]. Porto Alegre: Faculdade de Medicina, Universidade Federal do Rio Grande do Sul; 1995.

21. Gross JL, Eizirik DL, Kruter RH. Duração do diabete melito e complicações microangiopáticas. Rev Assoc Med Bras 1982; 28:| $40-2$.

Artigo recebido: 19/09/2003

Aceito para publicação: 02/04/2004 